\title{
Falta ou perda de resposta aos ant-TNFs. Qual o próximo passo
}

\section{Silvio Danese}

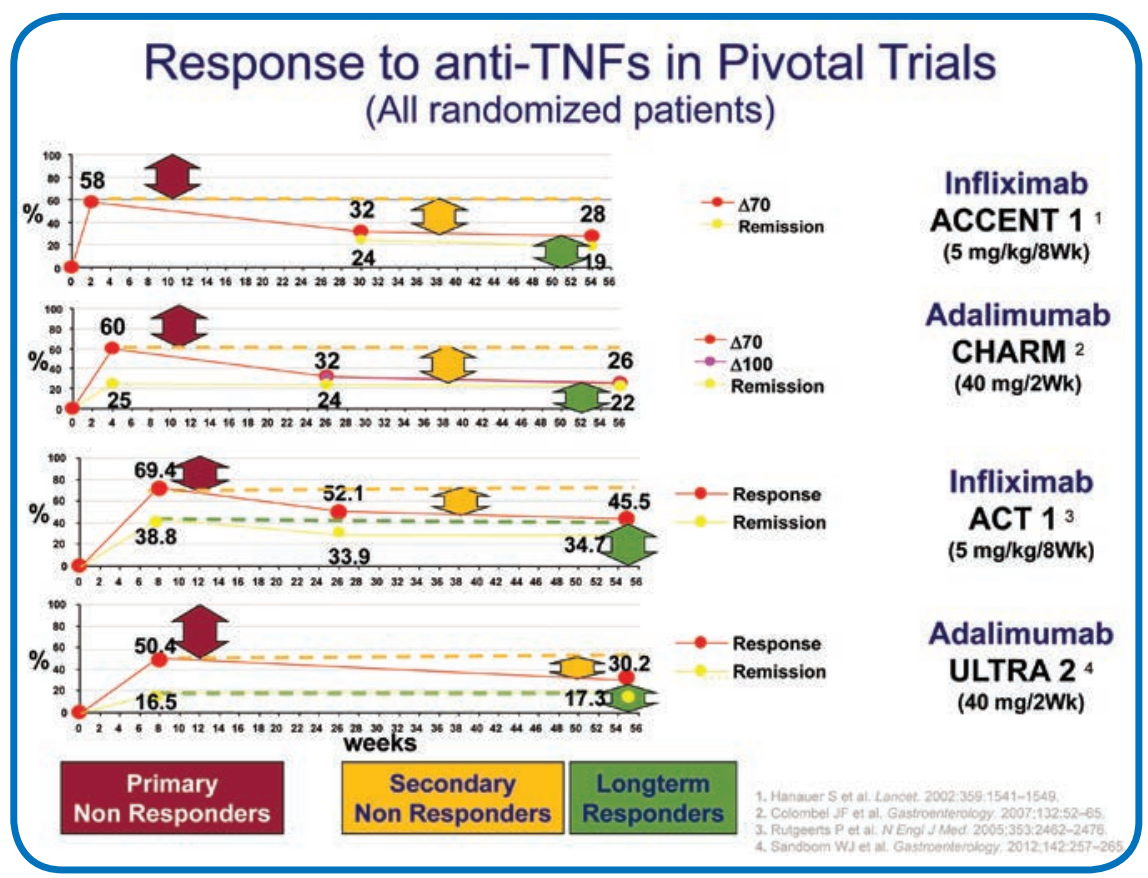




\begin{tabular}{|l|c|c|}
\hline \multicolumn{2}{|c|}{ Anti-TNF $\alpha$ for IBD: current knowledge } \\
\hline Type & Infliximab & Adalimumab \\
\hline Administration & chimeric & human \\
\hline Frequency & i.v. & s.c. \\
\hline Efficacy in luminal CD & Q8 & Q2 \\
\hline Efficacy in CS sparing & yes & yes \\
\hline Fistula healing & yes & yes \\
\hline Efficacy in UC & yes & yes (not licensed) \\
\hline MH & yes & yes \\
\hline Paediatric CD & yes & yes \\
\hline Paediatric UC & yes & yes \\
\hline Benefit/risk & yes & being studied \\
\hline & favorable & favorable \\
\hline
\end{tabular}

\section{Anti-TNF $\alpha$ for IBD: current knowledge}






\section{Optimization of anti-TNF treatment}

- Once anti-TNF has started, how to avoid and manage primary

failure?

Problem: how should we define primary failure?

- Broad range of "response intensity" (no clinical response, no clinical remission, no steroid-free clinical remission, no MH....)

\section{Primary non-response to anti-TNF alpha}

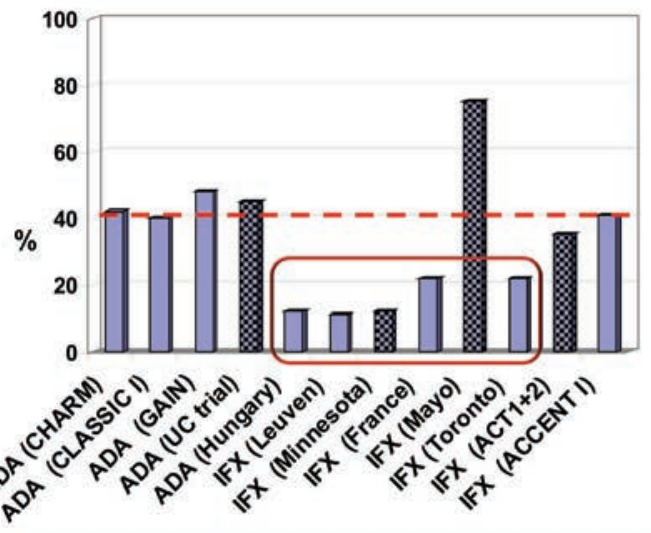

\section{PREDICTORS}

- Longer (>2 years) disease -

duration or small bowel disease

- Smoking-

(not in fistulizing disease)-

- Complicated phenotype-

- ANCAVASCA status (UC)-

- Normal CRP -

-Low $\mathrm{Hb}(<9.4)$ -

(single UC study)-

-Genetic-

(IBD5 mutation, FAS-L and -

Caspase 9 mutations, Non FC.

gammaRIIla genotype)

Ben Horin S, et al. Autoimm Rev 2014

\section{$20-40 \%$ of IBD patients in clinical trials}

$10-20 \%$ of IBD patients in clinical 'real life' series

Hanauer SB, et al. Lancet 2002; Rulgeerts P, et al.NEJM 2005; Hanaver S8, et al. Gastroenterology 2006; Colombel JF, et al. Gastroenterology 2007; Rudolph SJ, Dig Dis Soi 2008; A Ait W, et al. I8D 2009; Schnitzler F, et al. Gut 2009; Oussalah A, et al. AJG 2010; Kiss L.S, et al.APT 2011; Reinisch W, et al.Gut 2011: Ben Horin S, et al. Autoimm Rev 2014 
Primary failures with anti-TNF alpha

\section{Conclusion}

\section{Definition}

Prevention

- Patient selection

- Optimal induction regimen

- Consider IS and/or steroid co-treatment?

\section{Prediction?}

\section{Management:}

- Identification

- Consider all therapeutic options, including surgery

- Switch in-class

- Switch out-of-class (clinical trials; VDZ, UST)

- Other options in very difficult situations

\section{Optimization of anti-TNF treatment}

- Once anti-TNF has been successfully started, how to avoid or manage secondary failure and/or suboptimal treatment?

Definition (two key features):

1) Re-emerging symptoms appear in a patient whose disease was controlled by induction treatment

2) These symptoms are due to inflammatory process attributable to the underlying IBD 


\section{Mechanisms of worsening on anti-TNFs}

\section{Non-inflammatory mechanisms}

- IBS

- Fibrostenotic strictures

- Cancer

- Dietary (e.g. lactose)

- Miscellaneous (e.g. amyloidosis, SIBO, Bile salt diarrhoea)

Inflammatory mechanisms

Non-IBD related inflammation

- Infection

- Ischemis colitis

- Vasculitis

Un-controlled IBD inflammation (inadequate anti-TNF in serum)

- Loss of anti-TNF activity due to ADA

- Relentless TNF-mediated flare "consuming» the anti-TNF drug

- Loss of anti-TNF activity due to non-immune drug clearance

- Non-adherence to anti-TNF treatment

Un-controlled IBD inflammation (adequate anti-TNF in serum)

- Paradoxical exacerbation of inflammation by anti-TNFs

- Shift of disease pathway away from TNF to other mediators

\section{Loss of response to anti-TNF alpha}

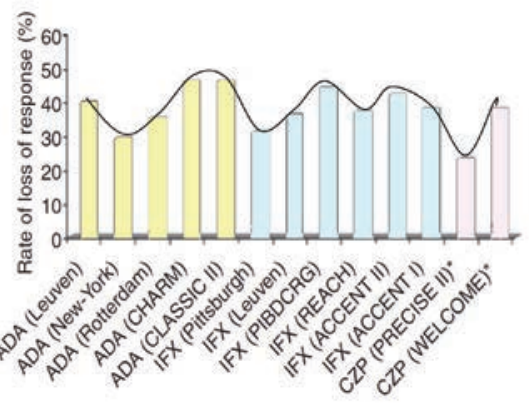

12-month Loss of response in case series and in RCTs ranges between $23 \%$ and $46 \%$

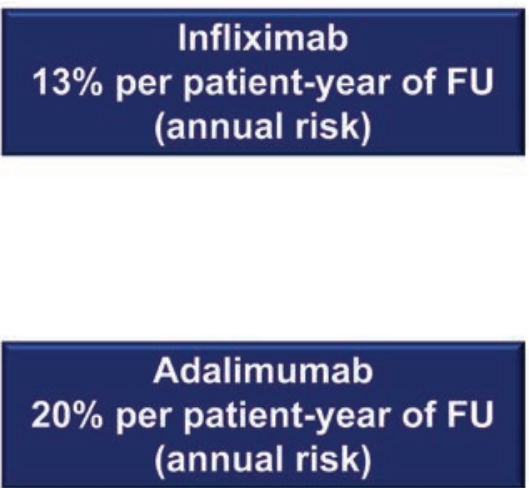




\section{Optimization of anti-TNF treatment to prevent loss of response}

- Chimeric / Humanized / Fully human biologics (No)

- Adequate induction doses and scheduled maintenance Tx (Yes)

- Concomitant Immunomodulators (¿Yes?)

- For IFX: better outcome for combo in IM naïve patients (SONIC, SUCCESS, Steroid-dependent UC)

- No RCT data with ADA

- No RCT in patients failing IMM drugs

- Conflicting results in retrospective cohorts (++/- -)

- No consensus on duration of combo therapy: for ever or 6 month?

- Consider adverse events

Vermeire S, et al. Gut 2007; Regueiro M, et al. IBD 2008; Hanauer SB, et al. CGH 2006; Baert F, et al. NEJM 2003; Farrel RJ, et al. Gastroenterology 2003; Schnitzler F, et al. Gut 2009; Sokol H, et al. Gut 2010; Reenaers C, et al APT 2012; Colombel JF, et al.

NEJM 2010; Panaccione R, et al. ECCO 2011; Armuzzi A, et al. IBD 2013; Kestens C, et al. CGH 2013; van der Valk ME, et al. Gut 2012; Baert F, et al. JCC 2013; Kiss LS, et al. APT 2011; Abraham NS, et al. CGH 2013;

\begin{tabular}{|l|l|}
\hline \multicolumn{2}{|c|}{ Factors affecting the PK of mAbs } \\
\hline Presence of ADAs & $\begin{array}{c}\text { Impact on PK } \\
\text { Threefold increase clearance } \\
\text { Worse clinical outcome }\end{array}$ \\
\hline Concomitant IS & $\begin{array}{c}\text { Reduces ADA formation } \\
\text { Increases serum mAbs } \\
\text { Decreases mAbs clearance } \\
\text { Better clinical outcome }\end{array}$ \\
\hline High baseline TNF & Increase mAbs clearance \\
\hline Low albumin & $\begin{array}{c}\text { Increase mAbs clearance } \\
\text { Worse clinical outcome }\end{array}$ \\
\hline High baseline CRP & Increase mAbs clearance \\
\hline Body size & High BMl increases clerance \\
\hline Gender & Males have higher clearance \\
\hline
\end{tabular}




\section{Managing loss of response - Dose intensification}
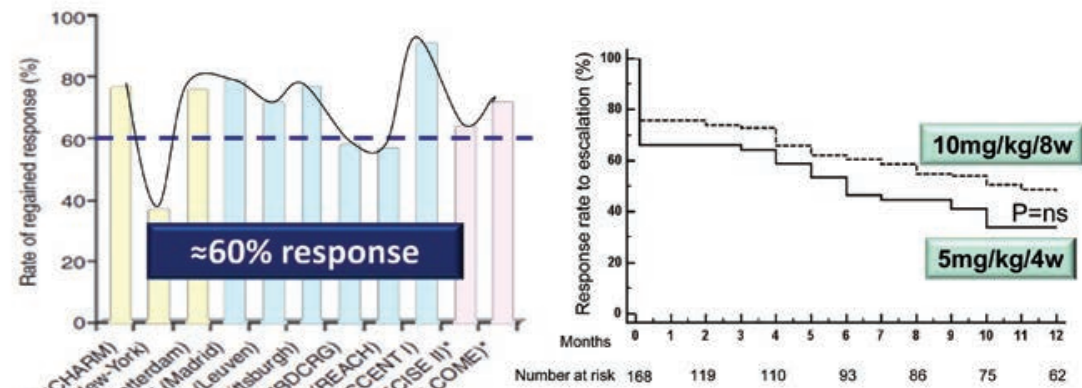

Combined sustained response: $47 \%$ at 12 months

\section{Anti-adhesion therapies for IBD}
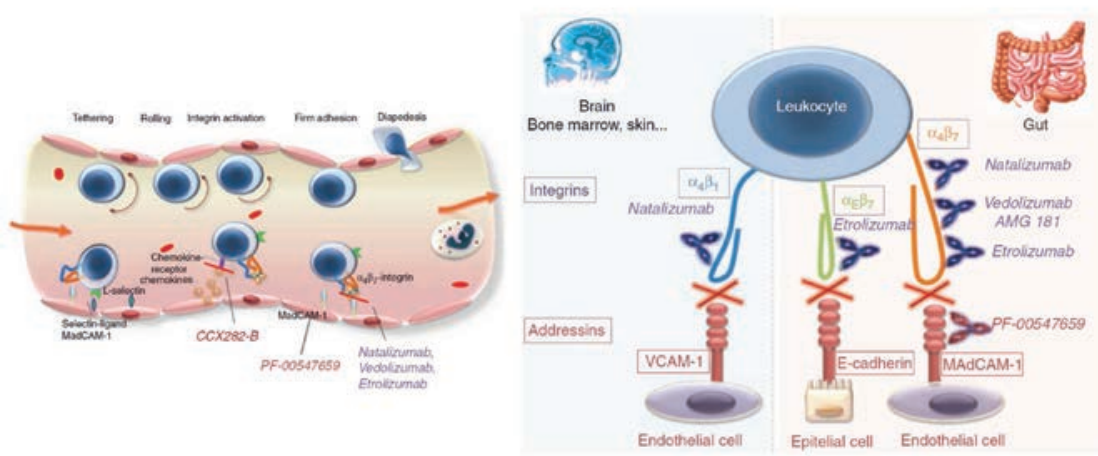


\section{Vedolizumab is a novel gut-selective anti-inflammatory biologic}

- Humanized mAb that binds exclusively to the $\alpha 4 \beta 7$ integrin heterodimer

- Does not bind to $\alpha 4 \beta 1$ or $\alpha E \beta 7$ integrin ${ }^{1}$

- Selective antagonist of $\alpha 4 \beta 7$ integrin

- Inhibits adhesion to MAdCAM-1 and fibronectin, but not VCAM-11

- Contains a mutated Fc region, preventing elicitation of ${ }^{2}$

- Complement-mediated cytotoxicity

- Antibody-dependent cellular cytotoxicity

- Cytokine release

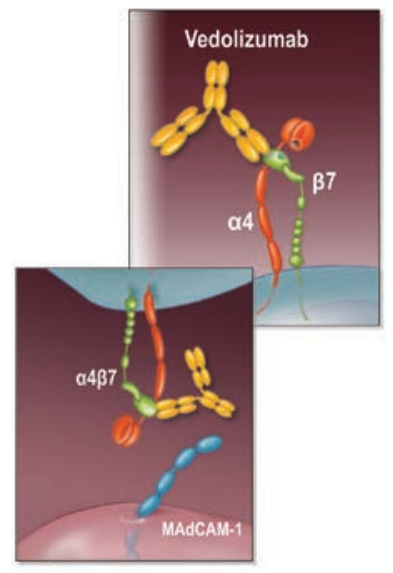

Fc, fragment crystallisable; mAb, monoclonal antibody ; VCAM-1, vascular cell adhesion molecule-1.

1. Soler D, et al. J Pharmacol Exp Ther 2009;330:864-75; 2. Data on file.

\section{Vedolizumab: clinical trial data}

\section{Phase 3 trials}

\begin{tabular}{|c|c|c|c|c|}
\hline Size & Arms & Primary endpoint(s) & $\begin{array}{l}\text { Trial } \\
\text { start }\end{array}$ & Completion \\
\hline \multicolumn{5}{|c|}{ Ulcerative colitis } \\
\hline $\begin{array}{l}826^{1} \\
\text { (GEMINI I) }\end{array}$ & $\begin{array}{l}\text { - Induction: vedolizumab or } \\
\text { placebo at weeks } 0,2,6 \\
\text { - Maintenance: vedolizumab Q4W } \\
\text { or Q8W, or placebo Q4W }\end{array}$ & $\begin{array}{l}\text { - Clinical response at Week } 6 \\
\text { - Clinical remission at Week } 52\end{array}$ & $\begin{array}{c}\text { Jan } \\
2009\end{array}$ & Jul 2012 \\
\hline \multicolumn{5}{|c|}{ Crohn's Disease } \\
\hline $\begin{array}{l}1,059^{2} \\
\text { (GEMINI II) }\end{array}$ & $\begin{array}{l}\text { - Induction: vedolizumab or } \\
\text { placebo at weeks } 0,2,6 \\
\text { - Maintenance: vedolizumab } Q 4 \mathrm{~W} \\
\text { or Q8W, or placebo } Q 4 \mathrm{~W}\end{array}$ & $\begin{array}{l}\text { - Clinical remission at Weeks } 6 \text { and } \\
52\end{array}$ & $\begin{array}{l}\text { Dec } \\
2008\end{array}$ & Oct 2012 \\
\hline $\begin{array}{l}416^{3} \\
\text { (GEMINI III) }\end{array}$ & $\begin{array}{l}\text { - Induction: vedolizumab or } \\
\text { placebo at weeks } 0,2,6\end{array}$ & $\begin{array}{l}\text { - Clinical remission in the } \\
\text { anti-TNF therapy subpopulation at } \\
\text { Week } 6\end{array}$ & $\begin{array}{l}\text { Nov } \\
2010\end{array}$ & Apr 2012 \\
\hline \multicolumn{5}{|l|}{ Safety } \\
\hline $\begin{array}{l}2,200^{4} \\
\text { (GEMINI LTS) }\end{array}$ & - Open-label vedolizumab Q4W & $\begin{array}{l}\text { - Safety, IBD-related events and } \\
\text { QoL up to week } 100\end{array}$ & $\begin{array}{l}\text { May } \\
2009\end{array}$ & Aug 2016 \\
\hline
\end{tabular}

1. Feagan et al. N Engl J Med 2013;369:699-710.

2. Sandborn WJ et al. N Engl J Med 2013;369:711-21.

3. Sands B et al. UEGW 2013, DDW 2014.

4. NCT00790933 clinicaltrials.gov. 


\section{GEMINI II: \\ vedolizumab in CD induction at Week 6}

\section{Induction ITT population}

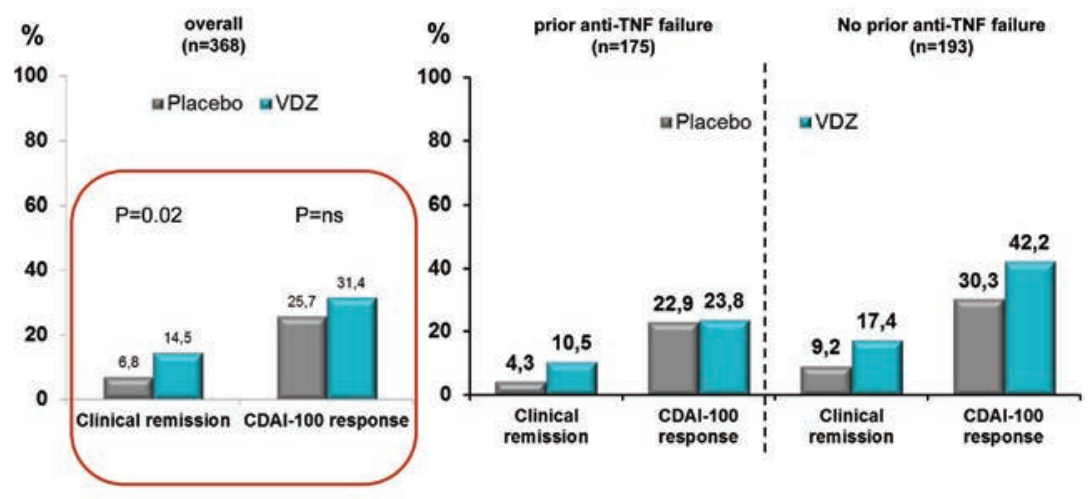

\section{GEMINI II:}

vedolizumab in CD maintenance at Week 52

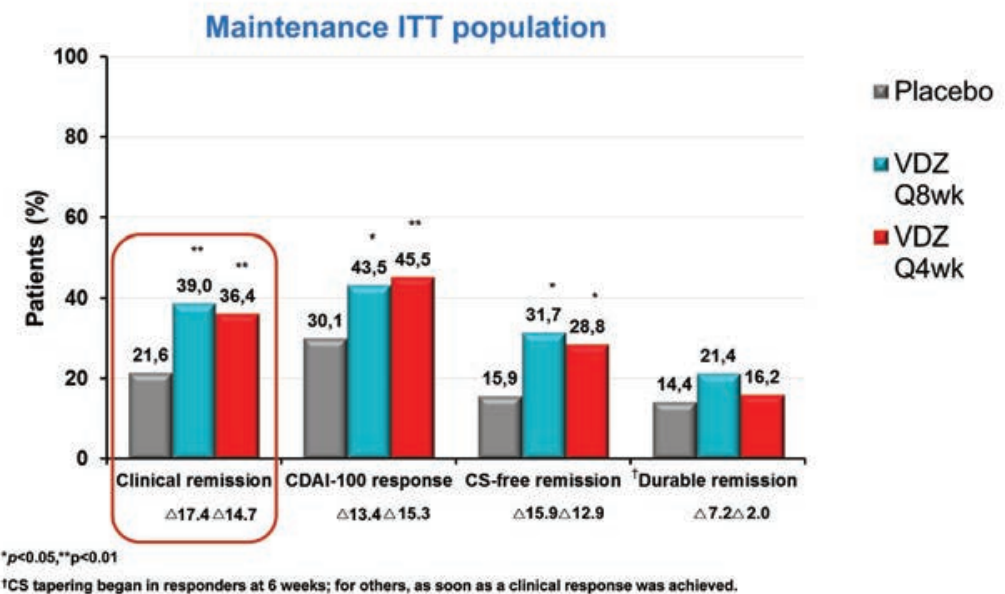

Sandborn WJ et al. N Engt J Med 2013;369:711-21 
GEMINI III:

vedolizumab induction therapy for CD and prior anti-TNF failure

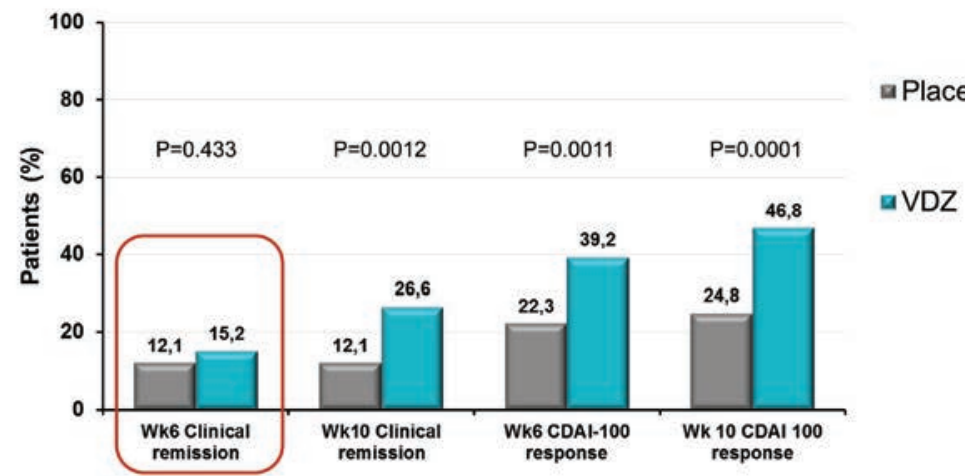

- Phase 3, multicentre, prospective, $\mathrm{RCT}(\mathrm{N}=315)$

- Randomised 3:2, patients received VDZ 300mg (IV) or PBO on days 1 \& 15

- Rates of AEs and serious AEs were similar between VDZ and PBO groups

\section{GEMINI II: adverse events affecting $\geq 5 \%$ of VDZ-treated patients during maintenance phase, safety population}

\begin{tabular}{lcc}
\hline Preferred term, N (\%) & $\begin{array}{c}\text { Placebo } \\
\mathrm{N}=301\end{array}$ & $\begin{array}{c}\mathrm{VDZ} \\
\mathrm{N}=814\end{array}$ \\
\hline $\mathrm{CD}$ & $65(22)$ & $164(20)$ \\
\hline Arthralgia & $40(13)$ & $110(14)$ \\
\hline Pyrexia & $40(13)$ & $103(13)$ \\
\hline Nasopharyngitis & $24(8)$ & $100(12)$ \\
\hline Headache & $47(16)$ & $97(12)$ \\
\hline Nausea & $30(10)$ & $90(11)$ \\
\hline Abdominal pain & $39(13)$ & $79(10)$ \\
\hline Upper respiratory tract infection & $17(6)$ & $54(7)$ \\
\hline Fatigue & $14(5)$ & $53(7)$ \\
\hline Vomiting & $23(8)$ & $49(6)$ \\
\hline Back pain & $12(4)$ & $38(5)$ \\
\hline Any serious AE & $46(15)$ & $199(24)$ \\
\hline
\end{tabular}

$\mathrm{CD}$, Crohn's disease.

${ }^{\circ} \mathrm{CD}(n=21$ [7\%] and 99 [12\%] for placebo and VDZ, respectively) and anal abscess ( $n=1[<1 \%]$ and16 [2\%]); all other serious AEs occurred in $s 1 \%$ of patients in each group. 


\section{GEMINI I: \\ vedolizumab induction therapy for UC at Week 6}

\section{Week 6 outcomes after 2-dose induction}

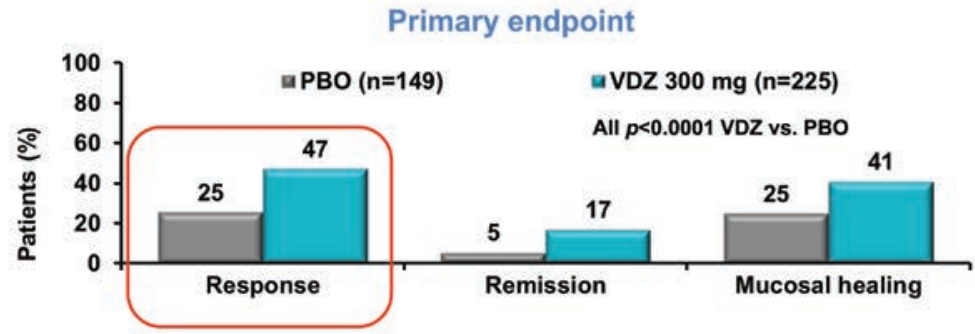

- Phase 3, multicentre, prospective, $\mathrm{RCT}(\mathrm{N}=374)$

- Randomised 3:2, patients received VDZ 300mg (IV) or PBO on days 1 \& 15

- Mod-to-severe active UC (Mayo 8.6, mean), despite conventional therapy

- UC diagnosis $\sim 6.4$ yrs, CS ( 54\%), IS ( $\sim 31 \%)$ and anti-TNF failures $(\sim 39 \%)$

- Rates of AEs and serious AEs were similar between VDZ and PBO groups

$A E$, adverse event; CS, corticosteroid; IS, immunosuppressant; RCT, randomised controlled trial.

\section{GEMINI I: \\ vedolizumab induction therapy for UC at Week 6}

\section{Week 6 outcomes after 2-dose induction by TNF antagonist-status}
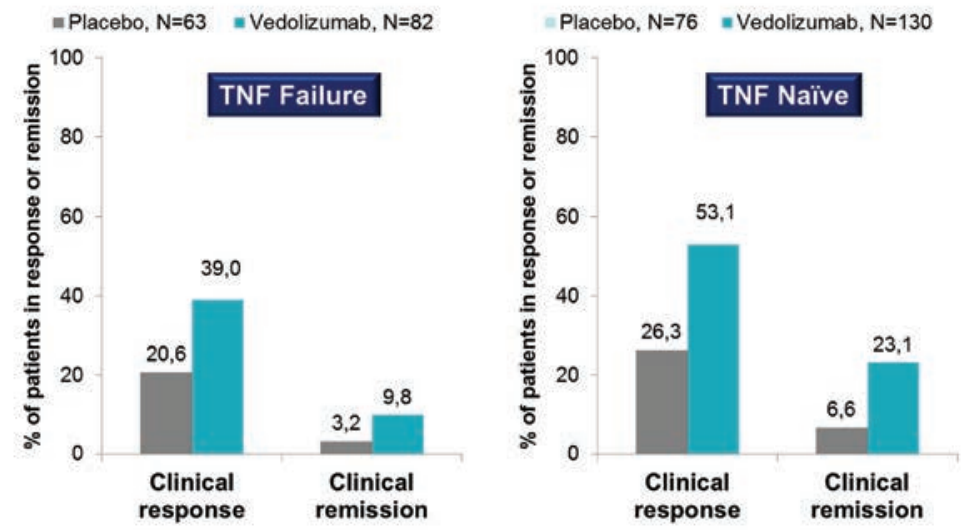


\section{GEMINI I: \\ vedolizumab in UC maintenance at Week 52}

\section{Primary and secondary efficacy endpoints}

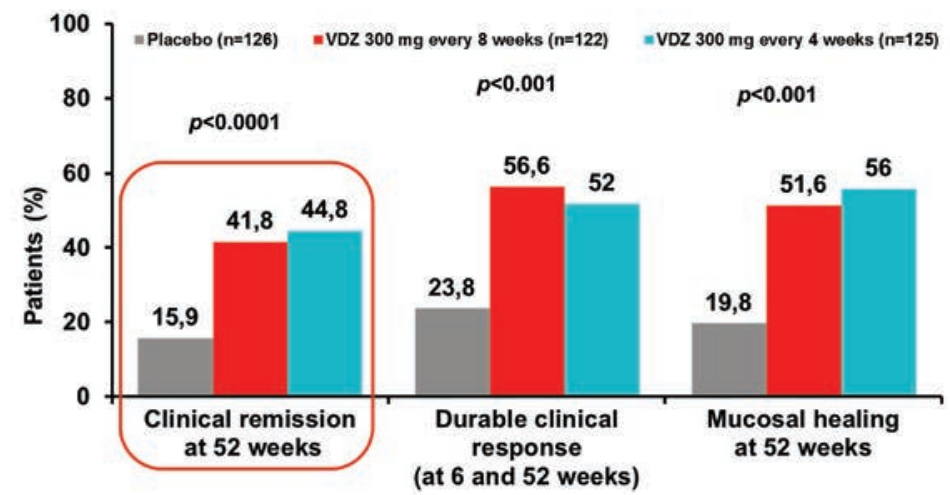

Feagan BG, et al. N Eng J Med 2013; 369:699-710.

\section{GEMINI I: \\ vedolizumab in UC maintenance at Week 52}

Clinical remission/durable clinical response by anti TNF antagonist-status
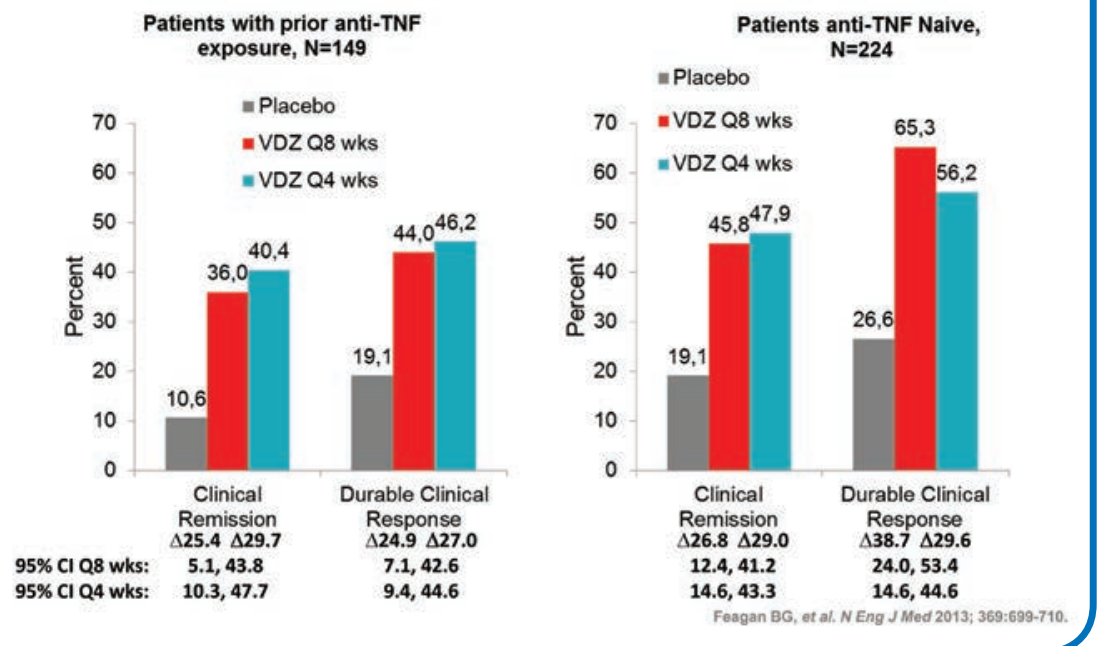


\section{GEMINI I:}

adverse events affecting $\geq 5 \%$ of VDZ-treated patients during maintenance phase, safety population

\begin{tabular}{l|c|c}
\hline Preferred term, N (\%) & $\begin{array}{c}\text { Placebo } \\
\text { N=275 }\end{array}$ & $\begin{array}{c}\text { VDZ } \\
\text { N=620 }\end{array}$ \\
\hline Colitis ulcerative & $58(21)$ & $97(16)$ \\
\hline Headache & $28(10)$ & $80(13)$ \\
\hline Nasopharyngitis & $26(9)$ & $80(13)$ \\
\hline Arthralgia & $25(9)$ & $56(9)$ \\
\hline Upper respiratory tract infection & $21(8)$ & $52(8)$ \\
\hline Nausea & $19(7)$ & $38(6)$ \\
\hline Cough & $13(5)$ & $36(6)$ \\
\hline Anemia & $16(6)$ & $35(6)$ \\
\hline Abdominal pain & $10(4)$ & $35(6)$ \\
\hline Fatigue & $10(4)$ & $33(5)$ \\
\hline Influenza & $6(2)$ & $30(5)$ \\
\hline Any serious AE & $37(13)$ & $77(12)$ \\
\hline
\end{tabular}

\section{PML risk assessment}

- >3000 patients treated with vedolizumab

$->2400$ with prior/concomitant immunosuppressant exposure

- >900 with over 24 months of exposure

$->50 \%$ presumed seropositive for $\mathrm{JC}$ virus

- No cases of PML have been observed in patients receiving vedolizumab 


\section{Phase II: safety and efficacy of etrolizumab (humanised anti- $\beta 7 \mathrm{mAb}$ ) for moderate-to-severe UC}

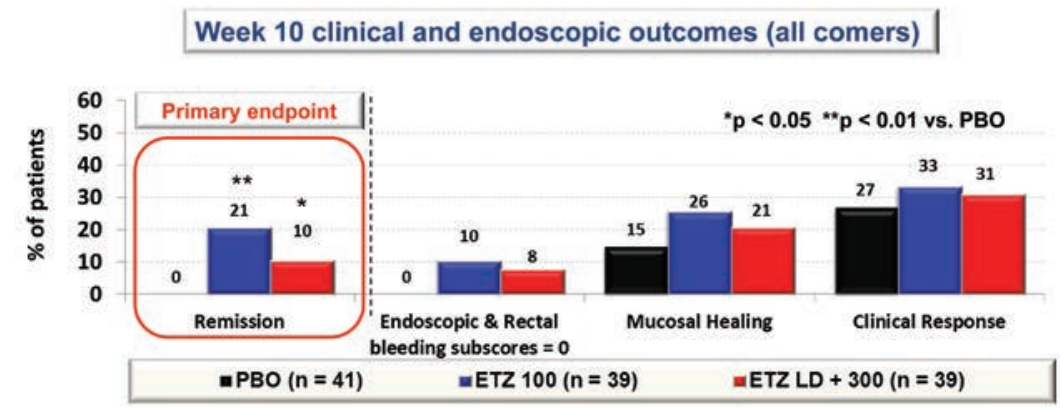

- Phase 2, multicentre, prospective, $\mathrm{RCT}(\mathrm{N}=119)$

- Randomised 1:1:1, patients received ETZ $100 \mathrm{mg} / \mathrm{mo}$ s.c. or $420 \mathrm{mg}$ loading dose between Week 0 \& 2 then $300 \mathrm{mg} / \mathrm{mo}$ s.c. or $\mathrm{PBO}$ for 3 doses

- Moderate-to-severe active UC: Mayo $\geq 5$, endoscopy subscore $\geq 2$ and RBS $\geq 1$

- UC diagnosis $\sim 9$ yrs, mean Mayo $\sim 9$, CS ( $\sim 42 \%)$, IS ( $38 \%)$, anti-TNF failures ( $60 \%)$

- Rates of AEs were comparable between ETZ and PBO groups

\section{Other promising targets}
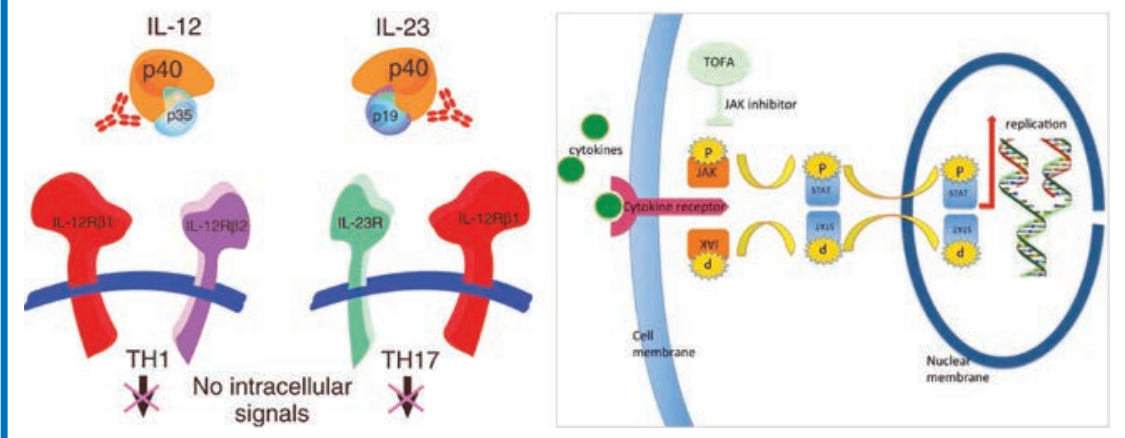


\section{Ustekinumab in moderate-to-severe Crohn's disease}

- Overall results

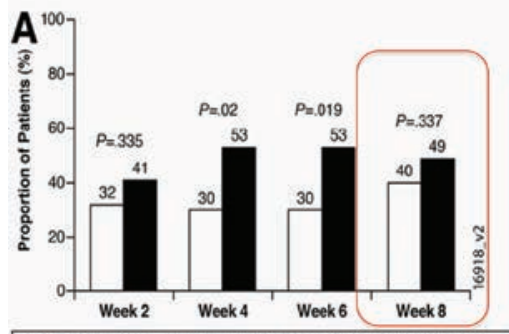

Combined subcutaneous and intravenous Placebo, Population $1(n=53)$ Combined subcutaneous and intravenous Ustekinumab, Population $1(n=51)$
- IFX experienced-patients

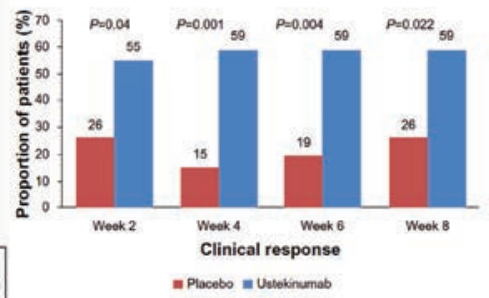

\section{CERTIFI: Ustekinumab in anti-TNF alpha resistant}

Crohn's Disease (Induction Phase)
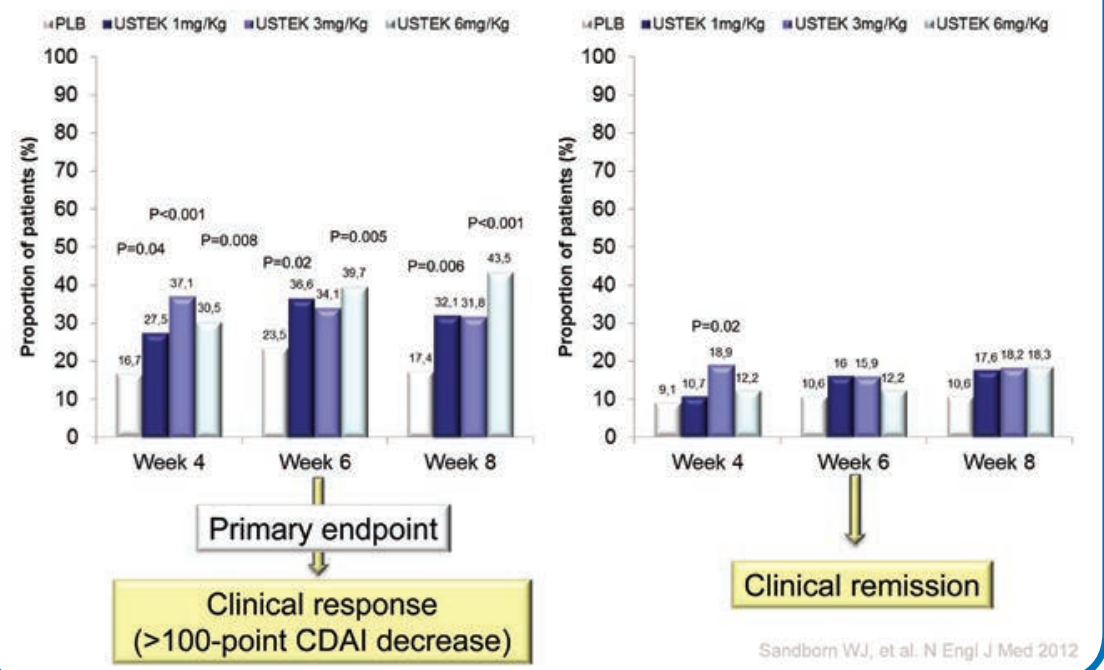


\section{CERTIFI: Ustekinumab in anti-TNF alpha resistant Crohn's Disease (Maintenance Phase)}

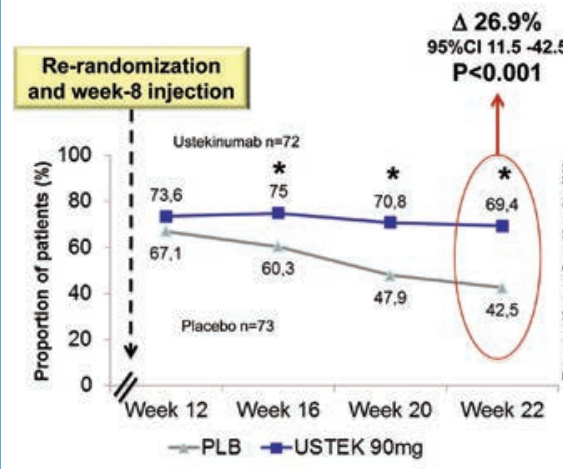

\section{Wk 22 Clinical response}

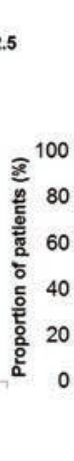

0

Week 12 Week 16 Week 20 Week 22

\#PLB -USTEK $90 \mathrm{mg}$
$\Delta 14.3 \%$

$95 \% \mathrm{Cl}$ 2.0.27.1

$P=0.03$

*: $p<0.05$

Wk 22 Clinical remission

\section{Phase III studies evaluationg ustekinumab in Crohn disease}

\begin{tabular}{|c|c|c|c|}
\hline$\overline{\text { Study }}$ & Description & $\begin{array}{l}\text { Study } \\
\text { end point }\end{array}$ & Status \\
\hline UNITI- ${ }^{34}$ & $\begin{array}{l}\text { Randomized placebo-controlled } \\
\text { trial evaluating ustekinumab in } \\
\text { moderate-to-severely active CD } \\
\text { with prior failure or intolerance } \\
\text { to anti-TNF } \alpha \text { therapy }\end{array}$ & $\begin{array}{l}\text { Clinical } \\
\text { response, } \\
\text { weck } 6\end{array}$ & $\begin{array}{l}\text { Completed, } \\
\text { results } \\
\text { pending }\end{array}$ \\
\hline UNITI- $2^{35}$ & $\begin{array}{l}\text { Randomized placebo-controlled } \\
\text { trial evaluating ustekinumab } \\
\text { in moderate-to-severely active } \\
C D \text { without prior failure } \\
\text { or intolerance to anti-TNF } \alpha \\
\text { therapy }\end{array}$ & $\begin{array}{l}\text { Clinical } \\
\text { response, } \\
\text { week } 6\end{array}$ & Recruiting \\
\hline IM-UNIT| ${ }^{36}$ & $\begin{array}{l}\text { Randomized placebo-controlled } \\
\text { trial evaluating safety and } \\
\text { efficacy of ustekinumab in CD } \\
\text { patients with clinical response } \\
\text { in UNITI-I or UNITI-2 }\end{array}$ & $\begin{array}{l}\text { Clinical } \\
\text { remission, } \\
\text { week } 44\end{array}$ & Recruiting \\
\hline
\end{tabular}

Tuskey A et al Clinical and Experimental Gasroenterology 2014;7:173-179 


\section{Tofacitinib, an oral Janus Kinase inhibitor, in active UC}
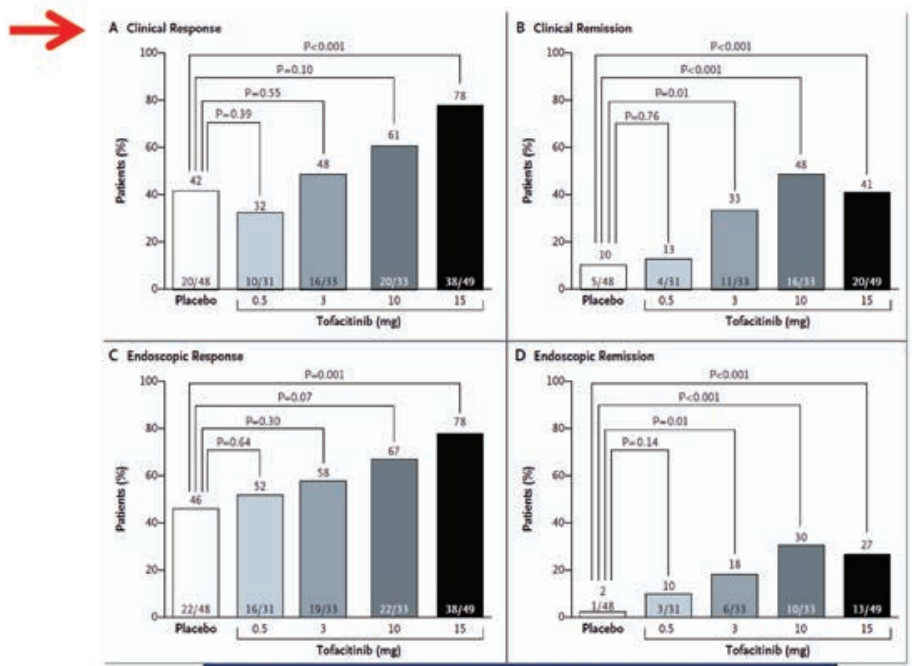

\section{4 active UC (Mayo $\geq 6$ ), 8-week treatment}

